\title{
2 PAVEMENT DAMAGE CRACK RECOGNITION 3 METHOD BASED ON HIGH-RESOLUTION 4 LINEAR ARRAY CAMERAS AND ADAPTIVE 5 LIFTING ALGORITHM
}

\author{
Yi Han ${ }^{1, *}$, Shiwei Tu ${ }^{1}$, Yanyu Yang ${ }^{1}$, Ke Lei ${ }^{2}$ and Chunlei Liu ${ }^{3}$ \\ 1 School of Automobile, Chang'an University, Xi'an 710064, China; hany@chd.edu.cn \\ 2 Shangtai Software (Shanghai) Co., Ltd., Shanghai 200020, China; 651225679@qq.com \\ 3 Department of Computer Science, Valdosta State University, Valdosta 31698, USA ; cliu@valdosta.edu \\ * Correspondence: hany@chd.edu.cn; Tel.: +86-29-82334143
}

\begin{abstract}
This paper proposes a crack recognition method based on high-resolution line array cameras and adaptive lifting algorithm. By defining the crack rate, this algorithm calculates the ratio of the crack area to the area of the entire collected image to characterize the damage extent of the current section. The algorithm first uses image preprocessing to reduce the image noise, then uses histogram equalization to enhance the feature of the crack region, divides the whole image into multiple sub-blocks, and extracts region features in the sub-block. At the same time, this algorithm defines related feature descriptors, and constructs weak classifiers according to each feature descriptor, and converts the weak classifiers into strong classifiers by using an adaptive lifting algorithm. Finally, this algorithm realizes the division of the crack regions. Experimental results show that the proposed algorithm can meet the actual needs and is better than other classical algorithms.
\end{abstract}

Keywords: line array cameras; pavement crack detection; feature analysis; adaptive lifting

\section{Introduction}

With the large-scale construction of high-grade pavement in China in recent years, the detection of pavement damage has become a very important task [1-4]. Currently, semi-automated testing vehicle equipment is widely adopted in the detection of pavement problems. This approach requires manual processing of offline data and fails to achieve full automatic detection of pavement damage [5-6].It also has many obvious drawbacks. Firstly, the results of manual processing may be affected by the subjectivity of manual detection and thus may not accurately and objectively reflect the real conditions of pavement [7-8]. Secondly, the efficiency of manual detection is usually very low, therefore consumes a lot of manpower. These drawbacks are extremely unfavorable to highway management and maintenance. Moreover, the recognition effect of automatic identification system is not satisfactory and there are still many problems [9-13]. The main causes of these problems are: (1) pavement interference factors, such as shading shadows, water stains, grease and so on; (2) complex road conditions, the lighting conditions of the pavement are different at different time periods, which is highly detrimental to our identification; (3) pavement damage 
types, including transverse cracks, longitudinal cracks, chaps, block fractures, etc.. In view of the current testing needs and situation, this paper proposes the use of image processing technology, combined with the adaptive lifting algorithm in machine learning to automatically identify the crack area on the road image [14-18]. The algorithm has high recognition rate and fast speed, and meanwhile can basically meet the actual needs.

\section{Materials and Methods}

\subsection{Image Acquisition}

In this paper, high-resolution linear array cameras, image capture cards, combined with integrated LED lights, industrial personal computer(IPC), optical encoder, GPS and other auxiliary devices are used to acquire and store real-time road images, and are integrated as a whole system in a commercial vehicle [19-22]. By contrast, we use line frequency of $140 \mathrm{kHz}$, a resolution of $4 \mathrm{k}$, the model for the Basler sprint-spL4096-140km CMOS linear array cameras to capture road images [23-26].

During the driving process of the vehicle, the photoelectric encoder rotates synchronously with the wheel to generate TTL(Transistor-Transistor Logic) pulse signals, which are processed by the data acquisition card and part of peripheral circuits [27-29]. The computer counts the pulses and converts them into mileage and speed information in real time. In this process, the pulse generated by the photoelectric encoder is modulated to generate a pulse trigger signal for the linear array cameras. When the left and right linear array cameras are triggered, the image of the road surface is collected [30-31]. After the image signal is processed by the image capture card via the Camera Link interface, the image is transmitted to IPC memory to complete the acquisition and storage of information on the road.

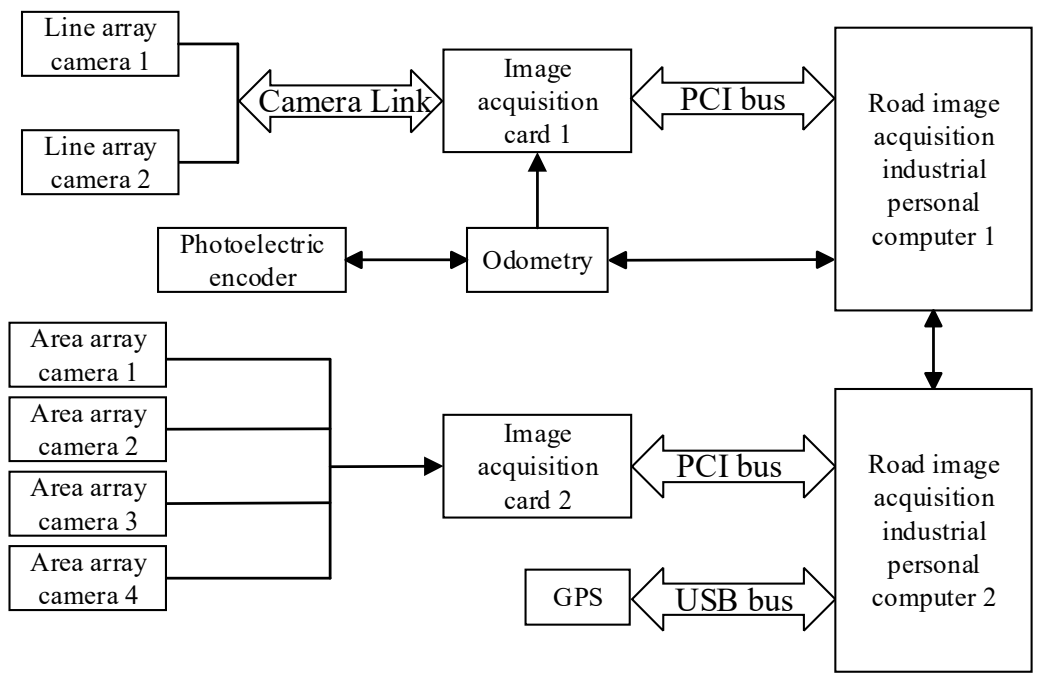

Figure 1. Image acquisition structure block diagram

After road images are obtained, road cracks can be identified through steps shown in Figure 2. 
64

65

66

67

68

69

70

71

72

73

74

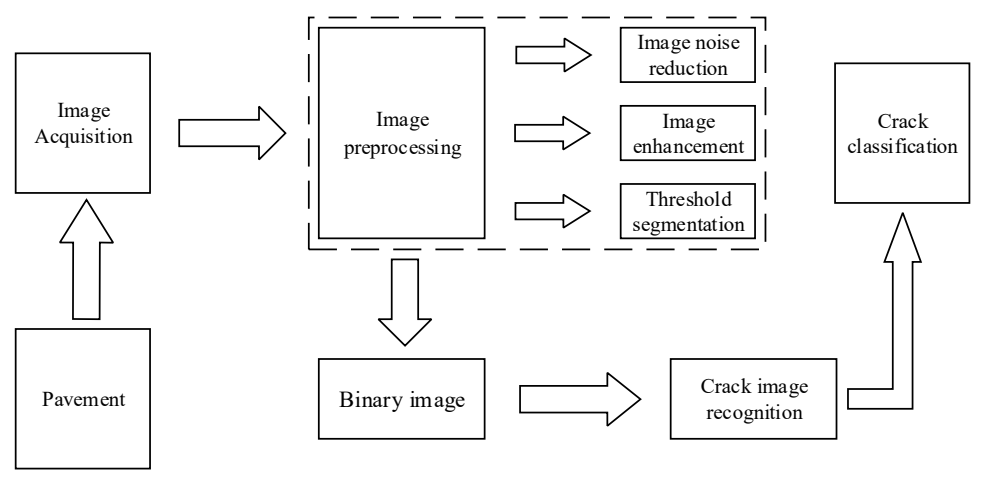

Figure 2. Operation flow chart

\subsection{Image preprocessing}

Before cracks in the captured image are identified, the image needs to be preprocessed because acquisition hardware and the natural lighting in the actual scene may inevitably introduce some interference and noise into the captured image [32-34]. In order to facilitate subsequent image processing, these unfavorable factors must be eliminated firstly. To avoid the obvious shortcoming of blurred image of the mean filter, we use the Gauss filter to reduce the image noise [35-38]. Our Gauss filter has a size of $5 \times 5$, and can be expressed as:

$$
F_{G S}=\frac{1}{273}\left[\begin{array}{ccccc}
1 & 4 & 7 & 4 & 1 \\
4 & 16 & 26 & 16 & 4 \\
7 & 26 & 41 & 26 & 7 \\
4 & 16 & 26 & 16 & 4 \\
1 & 4 & 7 & 4 & 1
\end{array}\right]
$$

The convolution of the Gauss filter and the original image $P_{o}$ produces the noise-reduced image $P_{d}$ [39].

$$
P_{d}=P_{o} \otimes F_{G S}
$$

After some noise is eliminated with the Gauss filter, the image can be further processed to enhance regional characteristics of the cracks to be identified and to weaken background features of the images [40]. The purpose of this process is to reduce the impact of the background information on the later recognition while preserving most of the crack information. We implement the image enhancement with histogram equalization, which converts the input image to hold the same pixel value in each gray scale [41-45]. This method can significantly enhance the contrast of the image. Assuming that the gray scale range of the captured image is $[0, L-1]$, the approximate probability of gray scale $r_{k}$ can be calculated as:

$$
p\left(r_{k}\right)=\frac{n_{k}}{N}, k=0,1, \ldots, L-1
$$


88 the numbers of all the pixels, and $L$ is for the number of gray scale $r_{k}$. Gray scale $r_{k}$ and the

89 probability of appearance of gray scale $p\left(r_{k}\right)$ can be expressed as the histogram of the original 90 image [46].

91 For gray images, the method of the enhancement of histogram equalization can be expressed 92 as [47]:

$$
\sum_{i=0}^{r_{k}} p(i)-\frac{f\left(r_{k}\right)}{L-1} \geq 0,0 \leq r_{k}<L
$$

94 Where

$$
f\left(r_{k}\right)=\sum_{j=0}^{r_{k}} u_{j}(0 \leq j<L)
$$

$$
\left\{\begin{array}{l}
u_{j} \geq 0 \\
\sum_{j=0}^{L-1} u_{j}<L
\end{array}(0 \leq j<L)\right.
$$

$97 f\left(r_{k}\right)\left(0 \leq r_{k}<L\right)$ stands for the mapping relationship between the pre-enhancement image gray

98 scale $r_{k}$ and the enhanced image gray scale $r_{k}{ }^{\prime}$.

99 By the following formula, the transformed gray scale allows the output histogram to be uniform 100 over the entire output gray scale range so that the contrast of the image can be increased.

$$
T\left(r_{k}\right)=\operatorname{round}\left((L-1) \sum_{j=0}^{k} p\left(r_{j}\right)\right)
$$
4. 
104

105

106

107

108

109

110

111

112

113

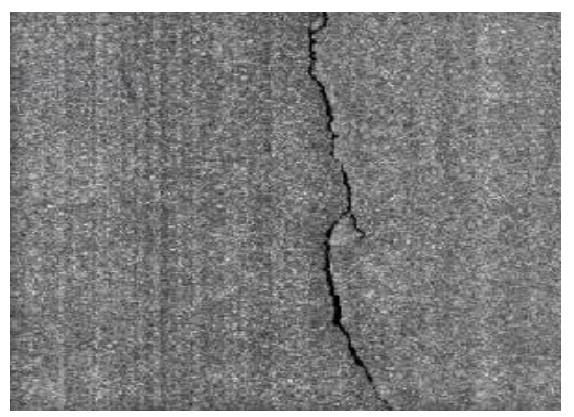

Figure 3. Original image

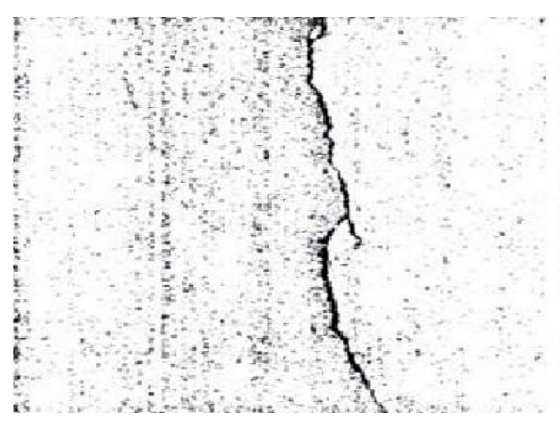

Figure 4. Image after preprocessing

Due to the complexity of the overall pavement condition, the gray value of cracks in different regions of the image varies greatly. Even if the whole image is segmented by the noise reduction with Gauss filter and the image enhancement with histogram equalization, the obtained results still can't meet the actual needs. Therefore, we divide the whole image into a plurality of sub-blocks, and the sub-block regions are separately subjected to threshold segmentation [48-49]. A collected 3024 $\times 2048$ pavement image is divided into $16 \times 16$ blocks, as shown in Figure 5 .

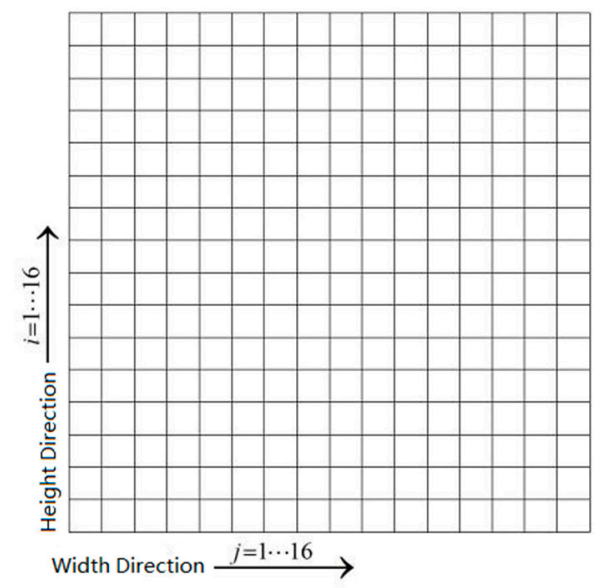

Figure 5. Regional block diagram

2.3. Feature selection

117 The 256 blocks fall into two categories, those containing cracks and those containing no cracks, 118 as shown in Figure 6. 
119

120

121

122

123

124

125

126

127

128

129

130

131

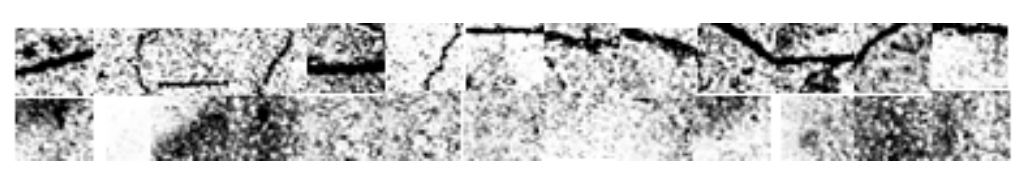

Figure 6. Image after dividing into blocks

Observation and analysis of a large number of image samples reveal that the target of pavement cracks has specific shape features that can be used to categorize the blocks. These shape features can be obtained by binarizing the segmented image with the maximum entropy method. The principle of maximum entropy method states that the entropy takes the maximum value when all events of the system are equally likely to occur [50-51].

The following is the process to use the maximum entropy method to calculate the threshold. For a gray image, assuming the range of the image gray values is $[0, L-1]$, and the minimum and maximum gray values are $V_{\min }$ and $V_{\max }$ respectively. According to the entropy formula, the entropy value corresponding to the gray value $t$ can be calculated as

$$
E(t)=\lg P_{t}\left(1-P_{t}\right)+\frac{H_{t}}{P_{t}}+\frac{H_{L}-H_{t}}{1-P_{t}}
$$

Where

$$
P_{t}=\sum_{i=0}^{t} p_{i}
$$

$$
H_{t}=-\sum_{i=0}^{t} p_{i} \lg p_{i}
$$

$$
H_{L}=-\sum_{i=0}^{L} p_{i} \lg p_{i}
$$

Here $p_{i}$ is the probability that the gray value $i$ appears, $P_{t}$ is the sum of the probability of

the gray values from 0 to $t, H_{t}$ is the sum of the entropy of the gray values from 0 to $t$, and $H_{L}$

137 is the entropy of the original image. The objective of our method is to find a proper value of $t$

138 between $V_{\min }$ and $V_{\max }$ to maximizes $E(t)$. The value of $t$ is the optimal threshold determined 139 by the maximum entropy method.

140 After the image process by using the threshold, there are still some discrete small particles in 141 the original picture. Therefore, four description values are designed as classification features of the 142 binary image for the obtained image area that is less than the threshold. 
143

144

145

146

147

148

149

150

151

152

153

where $\left\{x_{i}, y_{i}\right\}_{i=1}^{N}$ stands for training sample set and the corresponding mark.

(2) Initialize weights distribution of the training data set as

$$
D_{1}=\left(w_{1,1}, \ldots, w_{1, i}, \ldots w_{1, N}\right), w_{1, i}=\frac{1}{N}, i=1,2, \ldots N
$$

(3) For $m=1,2,3,4$, uses a training dataset with weight distribution $D_{m}$ for learning, and generates the basic classifier $G_{m}(x)$ [53]. In the meantime, calculates the error rate $e_{m}$ of 


$$
e_{m}=P\left(G_{m}\left(x_{i}\right) \neq y_{i}\right)=\sum_{G_{m}\left(x_{i}\right) \neq y_{i}} w_{m, i}
$$

$$
\alpha_{m}=\frac{1}{2} \lg \frac{1-e_{m}}{e_{m}}
$$

174 Where $w_{m, i}$ is the weight of the ith sample in round $m(m \in\{1,2,3,4\})$, and

$$
\sum_{i=1}^{N} w_{m, i}=1
$$

(4) Update the distribution of weights of the training data set.

$$
D_{m+1}=\left(w_{m+1,1}, \ldots, w_{m+1, i}, \ldots, w_{m+1, N}\right)
$$

$$
w_{m+1, i}=\frac{w_{m, i}}{Z_{m}} \exp \left(-\alpha_{m} y_{i} G_{m}\left(x_{i}\right)\right), i=1,2 \ldots, N
$$

179 Where $Z_{m}$ is the factor of normalization.

$$
Z_{m}=\sum_{i=1}^{N} w_{m, i} \exp \left(-\alpha_{m} y_{i} G_{m}\left(x_{i}\right)\right)
$$

(5) Construct the linear combination of the basic classifier and obtain the strong classifier that can determine whether the current sub-block image contains cracks.

$$
G(x)=\operatorname{sign}\left(\sum_{m=1}^{4} \alpha_{m} G_{m}(x)\right)
$$
as the ratio of the number of marked sub-blocks to the total number of sub-blocks [54]. The recognition results are shown in Figure 7.

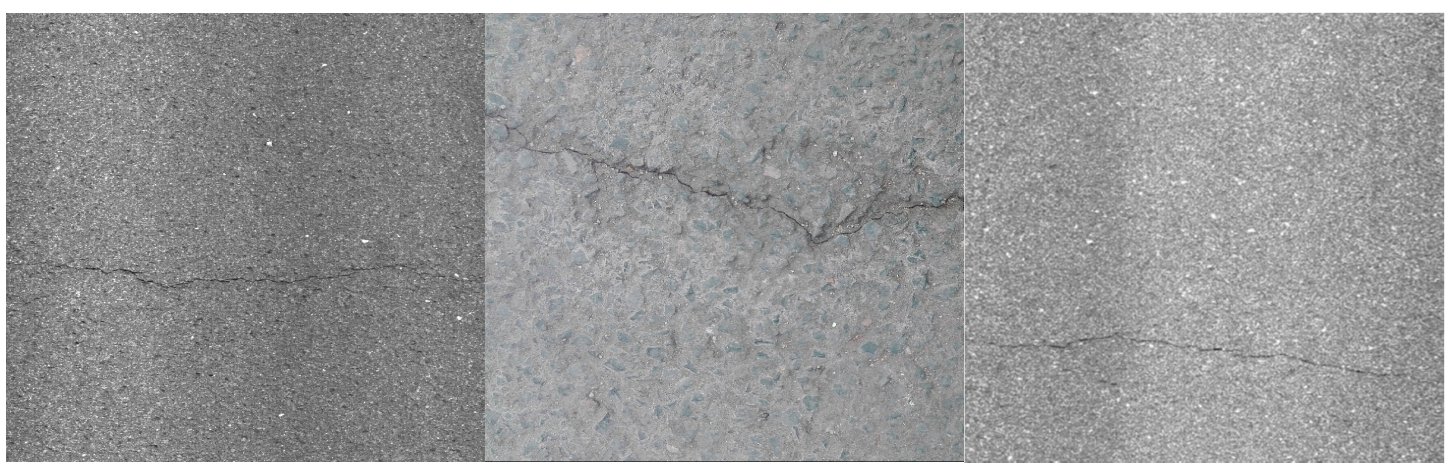


188

189

(a)

190

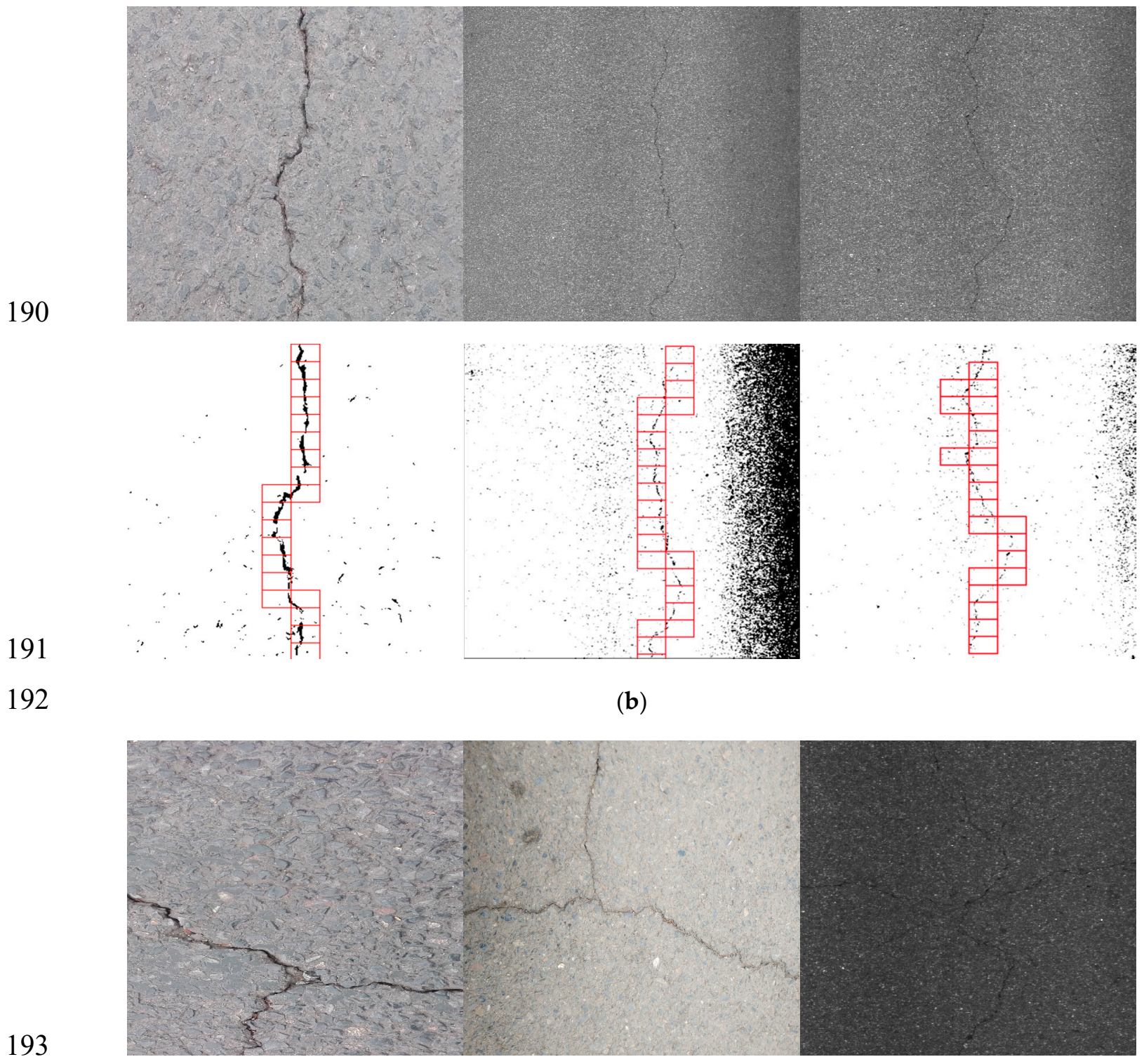

191

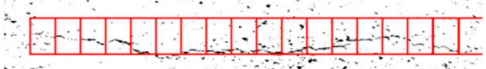
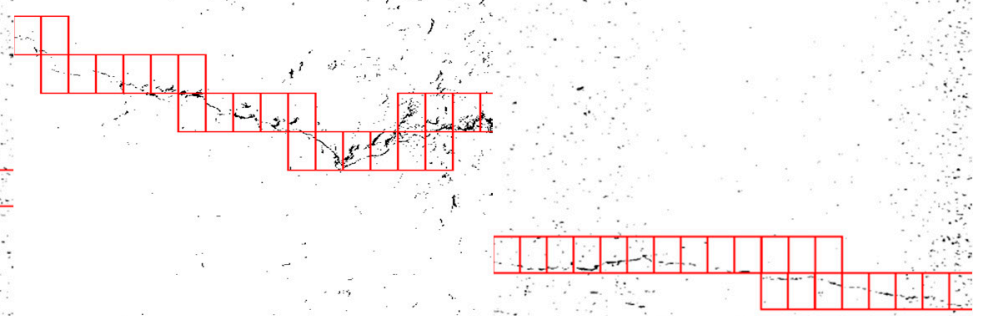
194

195

196

197

198

The overall algorithm is shown in Figure 8.

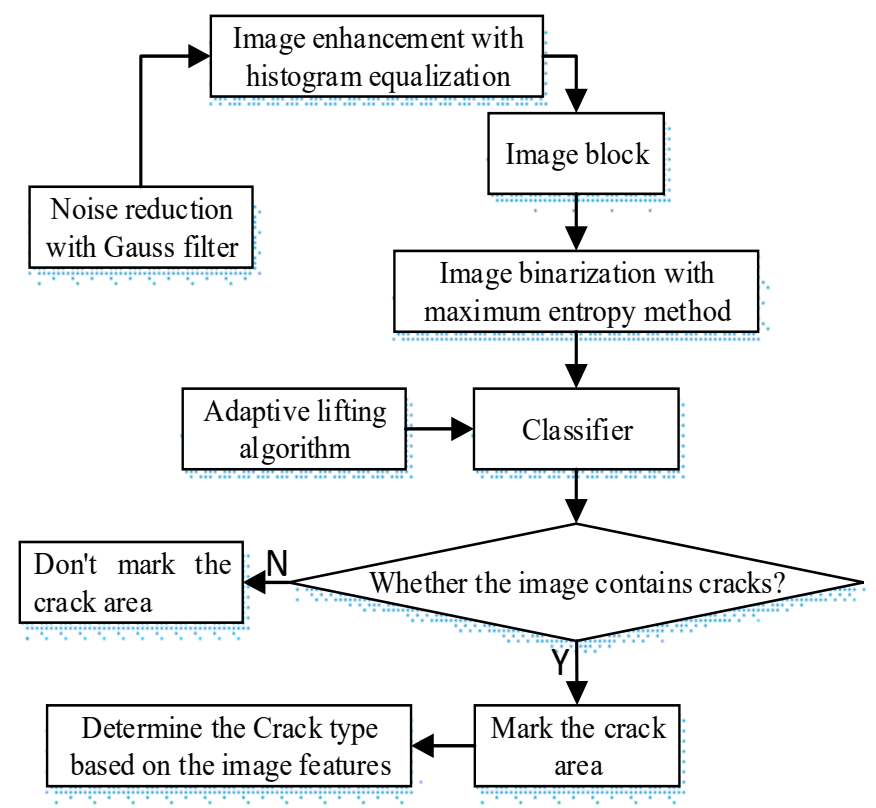

Figure 8. Flow diagram of algorithm

\section{Results}

To verify the effectiveness of this algorithm, a set of 1000 pavement damage pictures provided by a provincial highway bureau were used to perform a comparison experiment with manual detection, and two other commonly used algorithms: the minimal deviation algorithm and the OSTU algorithm. The experiment hardware environment is 2.40GHZ CPU, 8G memory IPC, and the software environment is VC2010. The experiment was repeated eight times and the results are shown below in Table 1. 


\begin{tabular}{llccc}
\hline Manual & Algorithm in \\
detection & $\begin{array}{c}\text { Minimal } \\
\text { deviation } \\
\text { algorithm }\end{array}$ & $\begin{array}{c}\text { OSTU } \\
\text { algorithm }\end{array}$ \\
\hline$\# 1$ & $4387 \mathrm{~ms}$ & $235 \mathrm{~ms}$ & $892 \mathrm{~ms}$ & $1233 \mathrm{~ms}$ \\
$\# 2$ & $3315 \mathrm{~ms}$ & $346 \mathrm{~ms}$ & $1123 \mathrm{~ms}$ & $1452 \mathrm{~ms}$ \\
$\# 3$ & $5789 \mathrm{~ms}$ & $532 \mathrm{~ms}$ & $965 \mathrm{~ms}$ & $1678 \mathrm{~ms}$ \\
$\# 4$ & $4310 \mathrm{~ms}$ & $490 \mathrm{~ms}$ & $1387 \mathrm{~ms}$ & $1783 \mathrm{~ms}$ \\
$\# 5$ & $3520 \mathrm{~ms}$ & $456 \mathrm{~ms}$ & $732 \mathrm{~ms}$ & $1653 \mathrm{~ms}$ \\
$\# 7$ & $5120 \mathrm{~ms}$ & $378 \mathrm{~ms}$ & $1232 \mathrm{~ms}$ & $1723 \mathrm{~ms}$ \\
$\# 8$ & $4760 \mathrm{~ms}$ & $612 \mathrm{~ms}$ & $934 \mathrm{~ms}$ & $1974 \mathrm{~ms}$ \\
& $4239 \mathrm{~ms}$ & $563 \mathrm{~ms}$ & $1365 \mathrm{~ms}$ & $1923 \mathrm{~ms}$
\end{tabular}

It can be seen that the detection speed of the algorithm in this paper is faster than that 212 manual detection and the other two commonly used algorithms. We also compared the accuracy of 213 the detection methods of the four algorithms. The results are shown in Figure 9.

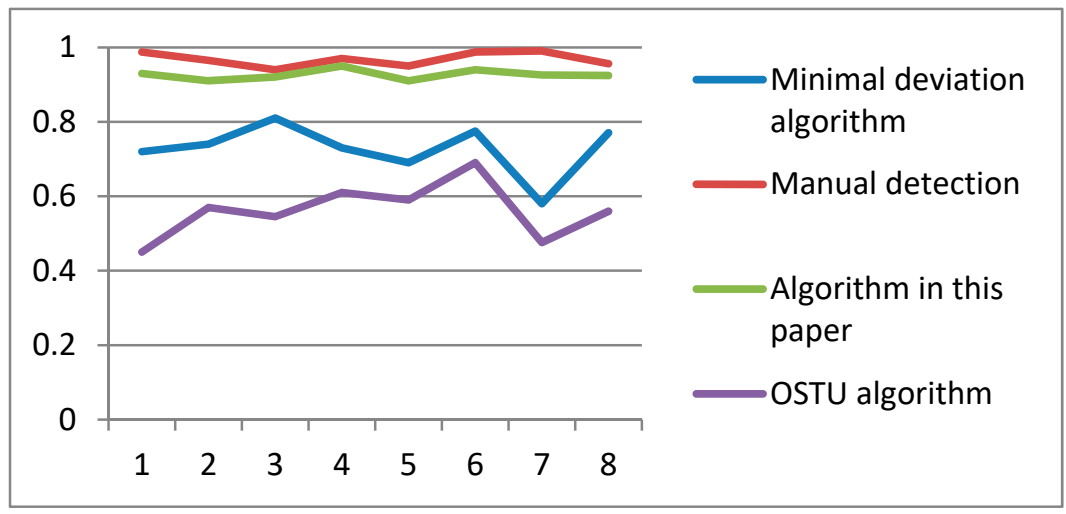

Figure 9. Accuracy of crack detection of the four methods

\section{4. Discussion}

217 From Figure 9, we can see that the accuracy of crack detection of the proposed algorithm is 218 slightly lower than manual detection, but far superior to the other two commonly used algorithms. 219 The accuracy of the algorithm in this paper can meet the detection accuracy requirements of the 220 actual pavement detection department.

\section{$221 \quad$ 5. Conclusions}

222 Through theoretical research and actual experiments, we can draw the following conclusions:

(1) Currently, the detection of pavement crack is mainly conducted with manual identification. On the other hand, there are still many problems with automatic recognition, such as slow identification, poor accuracy and so on. Therefore, this paper adopts the adaptive lifting algorithm for automatic pavement crack recognition. 
(2) Through image processing combined with adaptive lifting model in machine learning, we can calculate the ratio of the number of crack sub-blocks to the total number of image sub-blocks and use it to characterize the degree of crack damage in the current image. The results suggest that the speed and accuracy of recognition of our proposed algorithm can meet actual requirements.

(3) The current research mainly focuses on pavement cracks, but it cannot automatically detect other types of damage, such as bags, pits, and repair of cracks. In the future, our research goal should place on other types of damage pavement to further improve the automatic pavement damage recognition system.

Acknowledgments: The Project Sponsored by the International Science and Technology Cooperation and Exchange Projects of Shaanxi China (2016KW-063).

Author Contributions: Y.H. and C.L.L. conceived and designed the research; K.L., S.W.T. and Y.Y.Y. performed the experiments; S.W.T. and Y.Y.Y. wrote the manuscript; Y.Y.Y. and S.W.T. analyzed the data; C.L.L. and K.L. helped to design the comparison algorithm; and Y.H. helped to design image processing methods.

Conflicts of Interest: The authors declare no conflict of interests.

\section{References}

1. Hasni, H.; Alavi, A.H.; Jiao, P.; Lajnef, N.; Chatti, K.; Aono,K.; Chakrabartty, S. A new approach for damage detection in asphalt concrete pavements using battery-free wireless sensors with non-constant injection rates, Measurement, 2017, 110, 217-229.

2. Zhang, D.; Li, Q.; Chen, Y.; Cao, M.; He, L.; Zhang B. An efficient and reliable coarse-to-fine approach for asphalt pavement crack detection. Image and Vision Computing2017, 57, 130-146.

3. Ouma, Y.O.; Hahn, M. Pothole detection on asphalt pavements from 2D-colour pothole images using fuzzy c-means clustering and morphological reconstruction. Automation in Construction2017, 83, $196-211$.

4. Xu, X.; Peng, S.; Xia, Y.; Ji, W. The development of a multi-channel GPR system for roadbed damage detection. Microelectronics Journal2014, 45, 1542-1555.

5. Kapela, R.; Śniatała, P.; Turkot, A.; Rybarczyk, A.; Pożarycki, A.; Rydzewski, P.; Wyczałek, M.; Błoch A. Asphalt surfaced pavement cracks detection based on histograms of oriented gradients. 2015 22nd International Conference Mixed Design of Integrated Circuits \& Systems (MIXDES), Torun, 2015, pp. 579-584.

6. Li, J.; Zhang, Y.; Wang, L. Design and implementation of pavement crack detection system based on FPGA. The 27th Chinese Control and Decision Conference (2015 CCDC), Qingdao, 2015, pp. 5936-5941.

7. Krysiński, L.; Sudyka J. GPR abilities in investigation of the pavement transversal cracks. Journal of Applied Geophysics, 2013, 97, 27-36.

8. Hadjidemetriou, G.M.; Christodoulou, S.E.; Vela, P.A. Automated detection of pavement patches utilizing support vector machine classification. 2016 18th Mediterranean Electrotechnical Conference (MELECON), Lemesos, 2016, pp. 1-5.

9. Sun, L.; Qian, Z. Multi-scale wavelet transform filtering of non-uniform pavement surface image background for automated pavement distress identification. Measurement, 2016, 86, $26-40$.

10. Pereira, F.C.; Pereira, C.E. Embedded Image Processing Systems for Automatic Recognition of Cracks using UAVs. IFAC-PapersOnLine, 2015, 48, 16-21. 
11. Cheng, H.D.; Miyojim, M. Automatic pavement distress detection system. Information Sciences,1998, 108, 219-240,

12. Radopoulou, S.C.; Brilakis,I. Patch detection for pavement assessment. Automation in Construction, 2015, 53, 95-104,

13. María, V.G.; Mercedes, S.; Joaquín, M.S.;Arias, P. A semi-automatic processing and visualisation tool for ground-penetrating radar pavement thickness data. Automation in Construction,2014, 45, 42-49.

14. Mataei, B.; Nejad, F.M.; Zahedi, M.; Zakeri, H. Evaluation of pavement surface drainage using an automated image acquisition and processing system. Automation in Construction, 2018, 86, 240-255.

15. Chen, D.L.; Lu, Y.Y. Automatic detection of tunnel lining using image processing supported by terrestrial laser scanning technology. 2017 IEEE 2nd Information Technology, Networking, Electronic and Automation Control Conference (ITNEC), Chengdu, China, 2017, pp. 529-533.

16. Pablo, Q.B.; XArgüello, F.; Heras, D.B.; Benediktsson,J.A . Wavelet-Based Classification of Hyperspectral Images Using Extended Morphological Profiles on Graphics Processing Units. IEEE Journal of Selected Topics in Applied Earth Observations and Remote Sensing, vol. 8, no. 6, pp. 2962-2970, June 2015.

17. Mori, S. Deep architecture neural network-based real-time image processing for image-guided radiotherapy. Physica Medica,2017, 40, 79-87.

18. Kim, H.; Lee, J.; Ahn, E.; Cho, S.; Shin, M.; Sim, S.-H. Concrete Crack Identification Using a UAV Incorporating Hybrid Image Processing. Sensors 2017, 17, 2052.

19. Sheng, Q.; Wang, Q.; Xiao, H.; Wang, Q. Research on Geometric Calibration of Spaceborne Linear Array Whiskbroom Camera. Sensors 2018, 18, 247.

20. Zhang, P.; Arre, T.J.; Ide-Ektessabi, A. A line scan camera-based structure from motion for high-resolution 3D reconstruction. Journal of Cultural Heritage,2015, 16, 656-663.

21. Bugby, S.L.; Lees, J.E.; Bhatia, B.S.; Perkins, A.C. Characterisation of a high resolution small field of view portable gamma camera. Physica Medica, 2014, 30, 331-339.

22. Schwanke, U.; Shayduk, M.; Sulanke, K.-H.; Vorobiov, S.; Wischnewski, R. A versatile digital camera trigger for telescopes in the Cherenkov Telescope Array. Nuclear Instruments and Methods in Physics Research Section A: Accelerators, Spectrometers. Detectors and Associated Equipment, 2015, 782, 92-103.

23. Burri, S.; Bruschini, C.; Charbon, E. LinoSPAD: A Compact Linear SPAD Camera System with 64 FPGA-Based TDC Modules for Versatile 50 ps Resolution Time-Resolved Imaging. Instruments 2017, 1, 6.

24. Jennings, M.W.; Rutten, T.P.; Ottaway, D.J. Evaluation of the signal quality of an inexpensive CMOS camera towards imaging a high-resolution plastic scintillation detector array. Radiation Measurements, 2017, 104, 22-31.

25. Jiang, B.; Pan, Z.; Qiu, Y. Study on the key technologies of a high-speed CMOS camera. Optik International Journal for Light and Electron Optics, 2017, 129, 100-107.

26. Nemeth, B.; Piechocinski, M.S.; Cumming, D.R.S. High-resolution real-time ion-camera system using a CMOS-based chemical sensor array for proton imaging. Sensors and Actuators B: Chemical, 2017, 171$172,2012,747-752$.

27. Liu, R.; Liu, R. Signal acquisition technology for photoelectric encoder based on FPGA. Optik International Journal for Light and Electron Optics, 2016, 127, 9891-9895.

28. Ge, X.; Xie, Y. A TTL-controlled trigger generator with rise-time of nanosecond level. 2016 International Conference on Electromagnetics in Advanced Applications (ICEAA), Cairns, QLD, 2016, pp. 872-875.

29. Tiemann, I.; Spaeth, C.; Wallner, G.; Metz, G.; Israel, W.; Yamaryo, Y.; Shimomura, T.; Kubo, T.; Wakasa, T.; Morosawa, T.; Köning, R.; Flügge, J.; Bosse,H. An international length comparison using vacuum 
comparators and a photoelectric incremental encoder as transfer standard. Precision Engineering, 2008, $32,1-6$.

30. Chen, D.; Ni, J. Pulse compression-based improvement on the estimation accuracy of time interval between two trigger signals in light screen array. Optik, 2018, 158, 675-683.

31. Keerthana, K.; Shanmugaraja, M.; MaheshKannan, P. Comparison of conventional flip flops with pulse triggered generation using signal feed through technique. 2015 International Conference on Communications and Signal Processing (ICCSP), Melmaruvathur, 2015, pp. 0392-0397.

32. Wang, Y.; Fan, M.; Li, J.; Cui, Z.Sparse Weighted Constrained Energy Minimization for Accurate Remote Sensing Image Target Detection. Remote Sens. 2017, 9, 1190.

33. Solsona, S.P.; Maeder, M.; Tauler, R.; Juan, A.D. A new matching image preprocessing for image data fusion. Chemometrics and Intelligent Laboratory Systems, 2017, 164, 32-42.

34. Suhas, S.; Venugopal, C. R. MRI image preprocessing and noise removal technique using linear and nonlinear filters. 2017 International Conference on Electrical, Electronics, Communication, Computer, and Optimization Techniques (ICEECCOT), Mysuru, India, 2017, pp. 1-4.

35. Ji, X.; Yuan, P.; Shi, Z.; Li, J.; Wang, T.; Cao, S.; Gao, L. An effective self-adaptive mean filter for mixed noise. 2016 International Conference on Advanced Robotics and Mechatronics (ICARM), Macau, 2016, pp. 484-489.

36. Said, A.B.; Hadjidj, R.; Melkemi, K.E.; Foufou, S. Multispectral image denoising with optimized vector non-local mean filter. Digital Signal Processing, 2016, 58, 115-126.

37. Agneni, A. ON THE USE OF THE GAUSS FILTER IN MODAL PARAMETER ESTIMATION. Mechanical Systems and Signal Processing. 2000, 14, 193-204.

38. Krystek, M. A fast gauss filtering algorithm for roughness measurements. Precision Engineering, 1996, 19, 198-200.

39. Xiang, D.; Ban, Y.; Wang, W.; Tang, T.; Su, Y. Edge Detector for Polarimetric SAR Images Using SIRV Model and Gauss-Shaped Filter. IEEE Geoscience and Remote Sensing Letters, vol. 13, no. 11, pp. 1661-1665, Nov. 2016.

40. Wu, K.; Li, G.; Han, G.; Yang, H.; Liu, P. Color image detail enhancement based on quaternion guided filter. The Journal of China Universities of Posts and Telecommunications, 2017, 24, 40-50.

41. Chiu, C.-C.; Ting, C.-C. Contrast Enhancement Algorithm Based on Gap Adjustment for Histogram Equalization. Sensors 2016, 16, 936.

42. Ting, C.-C.; Wu, B.-F.; Chung, M.-L.; Chiu, C.-C.; Wu, Y.-C. Visual Contrast Enhancement Algorithm Based on Histogram Equalization. Sensors 2015, 15, 16981-16999.

43. Wang, Y.; Pan, Z. Image contrast enhancement using adjacent-blocks-based modification for local histogram equalization. Infrared Physics \& Technology, 2017, 86, 59-65.

44. Zuo, C.; Chen, Q.; Sui, X. Range Limited Bi-Histogram Equalization for image contrast enhancement. Optik - International Journal for Light and Electron Optics. 2013, 124, 425-431.

45. Rajpoot, P.S.; Chouksey, A. A Systematic Study of Well Known Histogram Equalization Based Image Contrast Enhancement Methods. 2015 International Conference on Computational Intelligence and Communication Networks (CICN), Jabalpur, 2015, pp. 242-245.

46. Qiao, X.; Bao, J.; Zhang, H.; Zeng, L.; Li, D. Underwater image quality enhancement of sea cucumbers based on improved histogram equalization and wavelet transform. Information Processing in Agriculture. 2017, 4 , 206-213. 
47. Wang, X.; Chen, L. An effective histogram modification scheme for image contrast enhancement. Signal Processing. Image Communication, 2017, 58, 187-198.

48. Bhandari, A.K.; Kumar, A.; Chaudhary, S.; Singh, G.K. A novel color image multilevel thresholding based segmentation using nature inspired optimization algorithms. Expert Systems with Applications, 2016, 63, 112-133.

49. Duan, J.; Zhang, Y.; Zheng, B. Lane line recognition algorithm based on threshold segmentation and continuity of lane line. 2016 2nd IEEE International Conference on Computer and Communications (ICCC), Chengdu, 2016, pp. 680-684.

50. Sun, R.; Zhao, C. Restoration of Space Object Images by Using A Maximum Entropy Method. Chinese Astronomy and Astrophysics, 2015, 39, 89-99.

51. Gzyl, H.; Horst, E.T.; Molina, G. Application of the method of maximum entropy in the mean to classification problems. Physica A: Statistical Mechanics and its Applications, 2015, 437, 101-108.

52. Liu, Y.; Cai, W.; Shao, X. Intelligent background correction using an adaptive lifting wavelet. Chemometrics and Intelligent Laboratory Systems, 2013, 125, 11-17.

53. Tong, Z.; Gao, J.; Zhang, H. Recognition, location, measurement, and 3D reconstruction of concealed cracks using convolutional neural networks. Construction and Building Materials, 2017, 146, 775-787.

54. Hasni, H.; Alavi, A.H.; Jiao, P.; Lajnef, N.; Chatti, K.; Aono, K.; Chakrabartty, S. A new approach for damage detection in asphalt concrete pavements using battery-free wireless sensors with non-constant injection rates. Measurement, 2017, 110, 217-229. 